\title{
Up for Review
}

$\mathbf{R}$ eaders who have glanced at the Journal's new instructions for authors may have noticed that metaanalysis is now classified as original research. Some journal editors consider meta-analyses to be clinical reviews. We disagree. We have moved it "above the fold," alongside other forms of original research, a position justified by the clinical, methodologic, and scientific challenges required of a well done meta-analysis. But where does that leave clinical reviews? Have they been moved down, to the "human interest section," with the soft stuff of an otherwise rigorous journal? Not at all.

In fact, we are committed to raising the quality and scientific merit of our clinical reviews, and intend to nurture, nag; and nudge our authors until they-and we--get it right. A bad review is aimless, biased, and potentially misleading; it can even be dangerous. ${ }^{1}$ But a good review is priceless. It creates knowledge. It informs as it changes behavior, and it manages to root in our brains or our pockets as research articles rarely do.

However, excellent clinical reviews are rare. ${ }^{2}$ Having sat idly by, hoping that the next month's mail would provide a consistently better crop of reviews than did the month's before, we now issue a challenge to JGIM readers and would-be clinical review authors. Send us high-quality reviews, reviews that address clearly stated questions, that are constructed with solid methods, and that offer insightful analyses and evidence-based recommendations, and we will do our best to have them published. What do we mean by high-quality reviews? Here are our thoughts.

First, clinical reviews should answer specific questions, particularly questions that practicing clinicians are likely to ask. Should diuretics be used in venous insufficiency? is an example of a single question, while the role of $H$. pylori antibody in determining the etiology, diagnosis, and management of dyspepsia is a series of issues that might be brought together in a single review. Sharp questions define the purpose of the review, and are appreciated by busy readers. Furthermore, they guide authors toward appropriate strategies for assembling and presenting information. State-of-the-art or update articles are valuable, and we encourage them, but we intend to hold fast to our first rule: every reviewer should have a specific purpose, stated clearly.

Second, clinical reviews should be constructed systematically $^{1.2}$ and should explicate the methods that were employed. The methods section of a clinical review should describe the system used to identify the relevant
Table 1

Advice for Developing High-quality Reviews

1. Address a question or questions that are clinically relevant and stated clearly

2. Describe methods and criteria for literature search and selection of articles

3. Analyze and synthesize data systematically with attention to rigor, leading to recommendations that are evidence-based

4. Present information using a structured abstract and effective algorithms, tables. and graphics

literature and the criteria for selecting articles for inclusion. The criteria should be consistent with the type of question addressed by the clinical review. For example, a clinical review of etiology would rely preferentially on well-done cohort or case-control studies, while a clinical review of treatment would ideally be based on randomized controlled trials. Citation of already published reviews or chapters from textbooks is acceptable, and may be useful in establishing a baseline of knowledge against which the new knowledge developed by the clinical reviewer can be judged. But it is difficult to see how an original clinical review, particularly one that provides a fresh look at a new question, can be developed principally from secondary sources.

Third, the information that is given should be analyzed and synthesized in a systematic way. The synthesis should account for any limitations or biases that may be present in the articles that are reviewed. There is no sharp line separating a rigorous, quantitative metaanalysis from a more qualitative clinical review. But regardless of the level of quantitative analysis they might include, clinical reviews should not be editorials. Their conclusions should follow directly from the data that are provided, and their final recommendations should be developed carefully, with attention given to the assumptions that are being made in extrapolating the review's conclusions to clinical practice.

And fourth, clinical reviews should be friendly to readers. They should be clear in their messages and considerate in their styles. Structured abstracts ${ }^{3}$ will be required, as much because of what they indicate about the review's organization as because of the service they provide for readers. Clinicians are busy. Time for reading is short, and memories even shorter. Clinical review authors should keep all this in mind and be creative in developing algorithms and presenting data using graphics and other memory aids that readers may wish to 
extract and carry with them. Toward that end, Table 1 summarizes our advice for developing high-quality clinical reviews.

We look forward to a journal that brings to its readers-clinicians and researchers alike-clinical reviews of the highest quality, reviews that will set standards for evidence-based primary care. At the same time, we appreciate that such reviews are not easily developed, and that many of our prospective clinical review authors may be inexperienced with the approach we describe. But we view this as a joint venture, with authors and editors working together to enhance the quality of JGIM's clinical reviews. The Journal is most assuredly up for re- view(s). - Jack ENDE, MD, Division of General Internal Medicine, Hospital of the University of Pennsylvania, Philadelphia, PA 19104-4283; and Cynthia D. Mulrow, MD, Ambulatory Care, Audie L. Murphy Memorial Veterans Hospital, San Antonio, TX

\section{REFERENCES}

1. Oxman AD. Checklists for review articles. BMJ. 1994;309:648-51.

2. Mulrow $\mathrm{CD}$. The medical review article: state of the science. Ann Intern Med. 1987;106:485-8.

3. Haynes RB, Mulrow CD, Huth EJ, Altman DG, Gardner MJ. More informative abstracts revisited. Ann Intern Med. 1990;113:69-76.

\section{Quality of AIDS Care in a Changing Health Care System}

$\mathbf{T}$ o date, few studies have been published that shed light on how well people with HIV-related illnesses fare in different kinds of care organizations and with different constellations of providers. In the issue of the Journal, Stone and colleagues,' using data from the Boston Health Study, explore correlates of satisfaction with ambulatory care for persons with AIDS. They provide one of the first comparisons of AIDS patients' perceptions of care in three organizationally distinct service settings: a public-hospital HIV clinic, a teaching-hospital group medical practice, and a staff-model health maintenance organization (HMO). Patients receiving their care at the public-hospital HIV clinic staffed by faculty attending physicians were significantly more likely than were those at the other two sites to rate the quality of care low. Perceptions of care among those AIDS patients treated at the HMO were comparable to those of the patients attending a general internal medicine group practice at a teaching hospital. These findings, if substantiated by subsequent research, are encouraging in view of the rapid growth of HMOs. There is no evidence from this study that people with AIDS treated in a staff-model HMO perceive their care to be of low quality. Indeed, the findings suggest that shifting AIDS patients out of hospital-based clinics and into HMOs might actually improve care.

The major finding of this study is that the patients who identified a primary nurse reported significantly higher quality of care scores than did the other patients. Moreover, the presence of an identifiable primary nurse was strongly predictive of greater patient satisfaction, independent of site of care. This is an important finding because surprisingly little research has focused on the contributions of nurses to AIDS care, despite their centrality in the provision of services. Prominent AIDS phy- sicians, in a national survey of what makes a great hospital, ranked nursing as the single most important component in an excellent inpatient AIDS service. ${ }^{2}$ In a single-site pilot study conducted in a teaching-hospital HIV clinic, we compared outcomes for ambulatory patients with HIV-related illnesses whose primary providers were nurse practitioners vs physicians. ${ }^{3}$ The patients of nurse practitioners reported significantly fewer problems with their care, and their functional levels were comparable to those of the physician-treated patients, even though the nurses patients were more severely ill on average.

There is a large research literature documenting the success of nurses in improving patient satisfaction, enhancing function, and reducing inappropriate utilization of emergency and inpatient services for persons with a wide range of chronic illnesses. ${ }^{4.5}$ Their success appears to be related to the centrality of caring (as compared to curing) to nurses and the nursing profession, and the high priority nurses have traditionally placed on maintenance or restoration of function in everyday living. ${ }^{6}$ Caring for persons with AIDS calls upon the entire range of physical, psychological, and social interventions that nurses are characteristically and, in many respects. singularly educated to provide. While nurses show some of the same fears of occupational transmission of HIV that remain widespread among health care workers, there is increasing evidence that nurses do not perceive caring for AIDS patients as hopeless, a perception that has been widespread among physicians. ${ }^{7}$

Several large-scale studies have interesting findings in this regard. Contrary to popular beliefs, nurses practicing on inpatient AIDS units who spend full time in AIDS care experience less burnout than do nurses practicing on general medical units. ${ }^{8}$ A national study of the 